\title{
Intracellular uptake of Pluronic copolymer: effects of the aggregation state
}

\author{
Md. Muniruzzaman ${ }^{\mathrm{a}}$, Alexander Marin ${ }^{\mathrm{a}}$, Yi Luo ${ }^{\mathrm{b}}$, Glenn D. Prestwich ${ }^{\mathrm{b}}$, William G. \\ Pitt ${ }^{\mathrm{c}}$, Ghaleb Husseini a,c, Natalya Y. Rapoport ${ }^{\mathrm{a}, *}$ \\ a20 S. 2030 E. Room 108, Department of Bioengineering, Center for Biopolymers on Interfaces, Uniersity of Utah, \\ Salt Lake City, UT 84112, USA \\ b Department of Medicinal Chemistry, Uniersity of Utah, Salt Lake City, UT 84112, USA \\ c Department of Chemical Engineering, Brigham Young Uniersity, Proo, UT 84602, USA Received 25 March 2001;
} accepted 20 November 2001

\begin{abstract}
The effect of the Pluronic P-105 aggregation state on its uptake by HL-60 cells was studied by flow cytometry, fluorescence spectroscopy, and confocal and fluorescence microscopy using a fluorescently labeled Pluronic P105. In the low concentration region below the critical micelle concentration (CMC), Pluronic uptake was proportional to the concentration in the incubation medium. The proportionality broke sharply above the CMC, revealing a less efficient intracellular uptake of Pluronic micelles than that of unimers. The data suggested that Pluronic micelles were internalized via fluid-phase endocytosis while unimers were internalized via diffusion through plasma membranes. Based on the above findings, the shielding effect of Pluronic micelles on drug intracellular uptake was explained.
\end{abstract}

Keywords: Pluronic micelles; HL-60 cells; Ultrasound; Intracellular uptake

\section{Introduction}

We reported earlier that drug encapsulation in polymeric micelles reduced intracellular drug uptake while ultrasonic irradiation of the cells enhanced uptake of micellar-encapsulated drugs [1-6]. These findings showed considerable promise for developing a technique of controlled and targeted delivery of drugs to solid tumors, the technique involving drug encapsulation in polymeric micelles followed by controlled release at the target site triggered by focused ultrasound [1-6]. These special polymeric micelles are formed by hydrophobic-hydrophilic block copolymers [1-14]. Their amphiphilic character, size $(10-30 \mathrm{~nm})$ and surface properties provide high drug loading capacity and long circulation time in the vascular system, which makes them attractive drug carriers $[7,8]$. The important advantage of polymeric micelles is a so-called enhanced penetration and retention (EPR) effect that provides a selective accumulation of micellar-encapsulated drugs in solid tumors [15-17]. The challenge to be met, however, is to ensure drug uptake by tumor cells. This would require the drug to be released from the micelles at the tumor site; upon release, drug is internalized by a regular mechanism specific for a particular drug/cell system. Another mechanism of drug uptake may be related to the enhanced endocytosis of the drug-comprising polymeric micelles. Ultrasonic irradiation is used in our study to activate both mechanisms. Our research provided evidence that under the action of ultrasound, drug release from micelles and drug uptake with micelles worked in concert[18,19]. 
The advantage of this technique is that ultrasound is non-invasive and can penetrate deep into the interior of the host. Ultrasound was shown to increase the extravasation of an MRI contrast agent [20]; for a micellar-encapsulated drug, this is expected to increase the selectivity of drug accumulation in the tumor. Ultrasound was shown to enhance permeability of cell membranes [21-23].

In our study, drugs were encapsulatedin Pluronic micelles. Pluronic is a triblock copolymer of poly(ethylene oxide) (PEO) and poly(propylene oxide) (PPO) of ABA type, where A stands for PEO and B stands for PPO. The hydrophobic central PPO blocks form micelle cores, whereas hydrophilic PEO blocks form the shells.

The effect of the ultrasound pulse duration on the intracellular uptake of Pluronic-encapsulated doxorubicin (DOX) [18] suggested that, at least partly, drug was taken up by the cells inside Pluronic micelles. In the present study, using confocal microscopy, flow cytometry, and fluorescence depletion from the incubation medium, we measured the intracellular uptake and intracellular distribution of fluorescently labeled Pluronic molecules from Pluronic solutions of various aggregation states. These experiments are essential as Pluronic copolymers in various aggregation states are anticipated for use as drug carriers. Pluronic unimers as drug carriers were thoroughly investigated by Kabanov and Alakhov [9-12]; the composition of Pluronic copolymers suggested by these authors is presently in phase I/IIa clinical trials.

In our studies $[1-6,18,19,25]$, we use dense Pluronic micelles rather than Pluronic unimers, exploring the shielding properties of micelles to target drugs to tumors while preventing unwanted drug interactions with healthy tissues. We activate the intracellular uptake of Pluronic micelle by ultrasonic irradiation.

\section{Experimental methods}

\subsection{Materials}

Pluronic P-105 with an average molecular weight of 6500, the number of monomeric units in PEO and PPO blocks being 37 and 56, respectively, was kindly supplied by the BASF Corporation (Mount Olive, NJ); Pluronic solution in PBS was used for the experiments. Dulbecco's PBS, pH 7.4 was purchased from Sigma and used as received.

\subsection{Synthesis of a fluorescently-labeled Pluronic}

$P 105$

About $28.7 \mathrm{mg}$ Pluronic-105 (Mw=6500) (8.8 mol of free $\mathrm{OH}$ group) was dissolved in $10 \mathrm{ml} \mathrm{DMF}$, and $1.97 \mathrm{mg}$ $(4.4 \mu \mathrm{mol})$ or $3.94 \mathrm{mg}(8.8 \mu \mathrm{mol})$ of 5 -(and -6)carboxy-27-dichlorofluorescein mixed isomers $(8.8 \mu \mathrm{mol})$ (Molecular Probes, Inc., Eugene, OR) in $5 \mathrm{ml}$ DMF was added. Next, $18.3 \mathrm{mg}$ dicycohexylcarbodimide $(8.8 \mu \mathrm{mol})$ and $2.2 \mathrm{mg}$ dimethylaminopyridine $(1.7 \mu \mathrm{mol})$ were added in solid form. The reaction mixture was stirred at room temperature for 3 days, and then quenched by $100 \mathrm{ml}$ ice-cold water. Gel permeation chromatography (GPC) indicated complete consumption of the initial fluorescent probe. The mixture was dialyzed against water for 7 days (three changes per day); the purity of fluorescently labeled Pluronic 105 was monitored by GPC. The product was lyophilized to obtain the dry powder of a fluorescently labeled Pluronic 105 with either a half or all hydroxyl groups labeled by a fluorescent probe. 
The reaction is schematically shown in Scheme

1.

2.3. Cells

HL-60 promyelocytic cells were kindly provided by Dr B.K. Murrey (Department of Microbiology, Brigham Young University, Provo, UT). They were cultured in RPMI-1640 medium supplemented with $20 \%$ fetal calf serum, $2 \mathrm{mM}$ L-glutamine, $0.2 \%$ sodium bicarbonate, and $50 \mathrm{~g} / \mathrm{ml}$ gentamicin at $37{ }^{\circ} \mathrm{C}$ in humidified air containing $5 \% \mathrm{CO}_{2}$.

\subsection{Cell incubation with a fluorescently-labeled Pluronic}

Cells were harvested, washed with PBS and resuspended in a solution of a fluorescently-labeled Pluronic in PBS; overall Pluronic concentration in a solution ranged from 0.0002 to $10 \mathrm{wt} . \%$. Two sets of experiments were made: (1) cells were incubated with progressively increasing concentrations of a fluorescently labeled Pluronic; (2) the concentration of a fluorescently labeled Pluronic in the incubation medium remained constant at either 10, 20, or $50 \mathrm{~g} / \mathrm{ml}$, and a non-labeled Pluronic was added to the incubation medium at concentrations below and above the CMC of Pluronic P-105 (based on our EPR data [3,24] and those of Alexandridis [14], for Pluronic P-105 at 37 ${ }^{\circ} \mathrm{C}$, the $\mathrm{CMC}$ for the formation of dense micelles with hydrophobic cores is close to $\left.1 \mathrm{wt} . \%\right)$. Upon incubation with Pluronic solution, cells were fixed with 3\% formalin and analyzed by flow cytometry, fluorescence microscopy and confocal microscopy. Pluronic uptake by the cells was also measured by fluorescence depletion from the incubation medium.

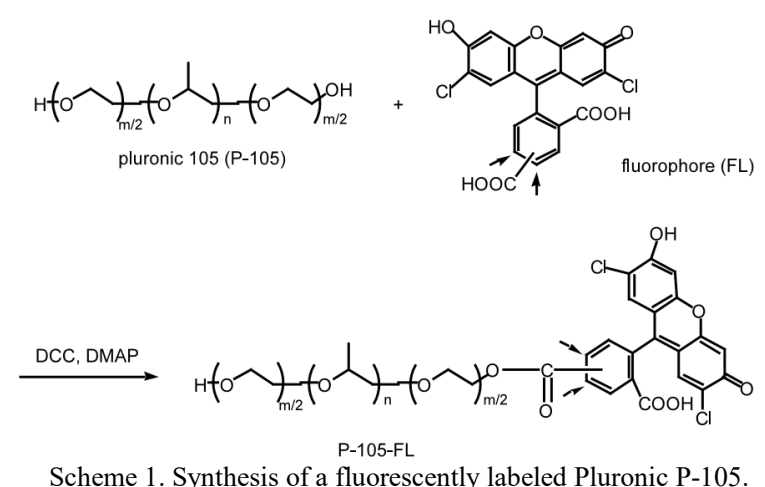

To discriminate between Pluronic adsorption onto cell surface, intracellular uptake via a simple diffusion or endocytosis, cell incubation with a fluorescently labeled Pluronic was performed either at 4 or at $37^{\circ} \mathrm{C}$, upon which the cells were fixed and analyzed by confocal microscopy before and after washing with PBS. Note that Pluronic P-105 does not form micelles at $4{ }^{\circ} \mathrm{C}[14]$; therefore, these experiments provide information on the adsorption or uptake for Pluronic unimers only.

\subsection{Measuring Pluronic P-105 uptake by the cells}

The intracellular uptake of labeled Pluronic was measured by fluorescence depletion from the incubation medium using a photon-counting spectrofluorimeter (ISS, model PC-1, Champaign, IL); the excitation wavelength was $488 \mathrm{~nm}$ and the emission wavelength was $550 \mathrm{~nm}$. Calibration experiments showed a linear dependence of 
fluorescence intensity of labeled Pluronic on its concentration in PBS in the concentration range used. The fluorescence intensity of tested solutions was compared with that of a standard labeled Pluronic solution of 1.0 $\mathrm{g} / \mathrm{ml}$. The cell number was counted with a hemacytometer and the amount of the internalized Pluronic was normalized to the cell number. When the mixtures of a labeled and unlabeled Pluronic were used, we assumed in our calculations presented in Table 2 that a labeled Pluronic was included into the

Scheme 1. Synthesis of a fluorescently labeled Pluronic P-105.

aggregates formed by unlabeled Pluronic and, therefore, cells did not distinguish between the labeled and unlabeled molecules in terms of the internalization; the overall Pluronic uptake was calculated taking into consideration a dilution factor:

$$
U_{\mathrm{t}}=U_{1} \times \frac{C_{\mathrm{t}}}{C_{1}}
$$

where $U_{\mathrm{t}}$ is the total uptake, $U_{\mathrm{l}}$ is the uptake of a fluorescently labeled Pluronic, $C_{\mathrm{t}}$ is total final Pluronic concentration in the incubation medium, and $C_{1}$ is the final concentration of the labeled

Table 1

Pluronic intracellular uptake at 4 and $37^{\circ} \mathrm{C}$ measured by fluorescence depletion from the incubation medium and by flow cytometry

Concentration of the unlabeled Pluronic Uptake of a fluorescently labeled Pluronic P-105 (wt.\%) measured by depletion from the incubation medium (ng per $10^{6}$ cells)

$4{ }^{\circ} \mathrm{C}$ $37{ }^{\circ} \mathrm{C}$

15.55 (unimodal) $\mathrm{n} / \mathrm{m}^{*}$

$\mathrm{n} / \mathrm{m}^{*}$
Geometrical mean fluorescence of the cells (arb.u.)

\begin{tabular}{|c|c|c|c|c|c|}
\hline & & & \\
\hline & & $4^{\circ} \mathrm{C}$ & $37^{\circ} \mathrm{C}$ & $4{ }^{\circ} \mathrm{C}$ & $37^{\circ} \mathrm{C}$ \\
\hline 0 (PBS) 175 & 100 & 7.55 (bimodal) & 15.55 (unimodal) & & \\
\hline 10 & & 16 & $\mathrm{n} / \mathrm{m}^{*}$ & 4.25 & 5.04 \\
\hline
\end{tabular}

Concentration of a labeled Pluronic P-105 in the incubation medium 0.0001\%; *non measurable.

Table 2

Pluronic intracellular uptake and partition coefficient as a function of the overall final Pluronic concentration in the incubation medium

\begin{tabular}{|c|c|c|c|}
\hline $\begin{array}{l}\text { Total initial Pluronic } \\
\text { concentration }(\%)\end{array}$ & $\begin{array}{l}\text { Uptake of a fluorescently labeled Pluronic } \\
\text { (g per } 10^{6} \text { cells) }\end{array}$ & $\begin{array}{l}\text { Total uptake of Pluronic } \\
\text { (g per } 10^{6} \text { cells) }\end{array}$ & $K_{\mathrm{eq}}$ \\
\hline 0.0016 & 1.55 & 1.55 & 32 \\
\hline 0.012 & 0.61 & 4.57 & 13 \\
\hline 0.102 & 0.18 & 11.0 & 3.8 \\
\hline
\end{tabular}

Concentration of a labeled Pluronic in the incubation medium 0.0016 wt.\%.

Pluronic. Partition coefficient $K_{\text {eq }}$ was calculated as $K_{\text {eq }}=U_{\mathrm{t}} / C_{\mathrm{t}}$. 


\subsection{Flow cytometry}

Fluorescence histograms were recorded with a Facscan (Beckton Dickinson) flow cytometer and analyzed using a CELLQUEST software supplied by the manufacturer. A minimum of 10000 events was analyzed to generate each histogram. To prevent Pluronic efflux from the cells upon washing, cell were injected into the flow cytometer in the incubation medium.

\subsection{Fluorescence microscopy/confocal microscopy}

The cells incubated with a fluorescently labeled Pluronic were first fixed with $3 \%$ formalin, then washed by PBS containing 3\% formalin, sealed on glass slides and visualized at $100 \times$ magnification by fluorescence microscopy with 527-552 nm excitation and 577-632 nm emission wavelengths (Eclipse E800, Nikon). When concentrations of a fluorescently labeled Pluronic in the incubation medium exceed $0.02 \%$, cells acquire a very high fluorescence, which complicates fluorescence imaging. To solve this problem, mixtures of a labeled and unlabeled Pluronic were used in these experiments, with the concentration of a labeled Pluronic being $0.001,0.002$, or $0.01 \%$, depending on the technique used, while the concentration of the unlabeled Pluronic varied in the range of $0.001-10 \%$. Confocal imagings were made with LSM 10 (ZEISS, Germany) instrument.

\section{Results and discussion}

\subsection{Pluronic intracellular uptake below and above the CMC}

Examples of flow cytometry histograms of the cells incubated at $37{ }^{\circ} \mathrm{C}$ with progressively increasing concentrations of a labeled Pluronic P105 are shown in Fig. 1. Confocal microscopy indicated that Pluronic molecules were internalized rather than absorbed onto the cell surface (Fig. 2).
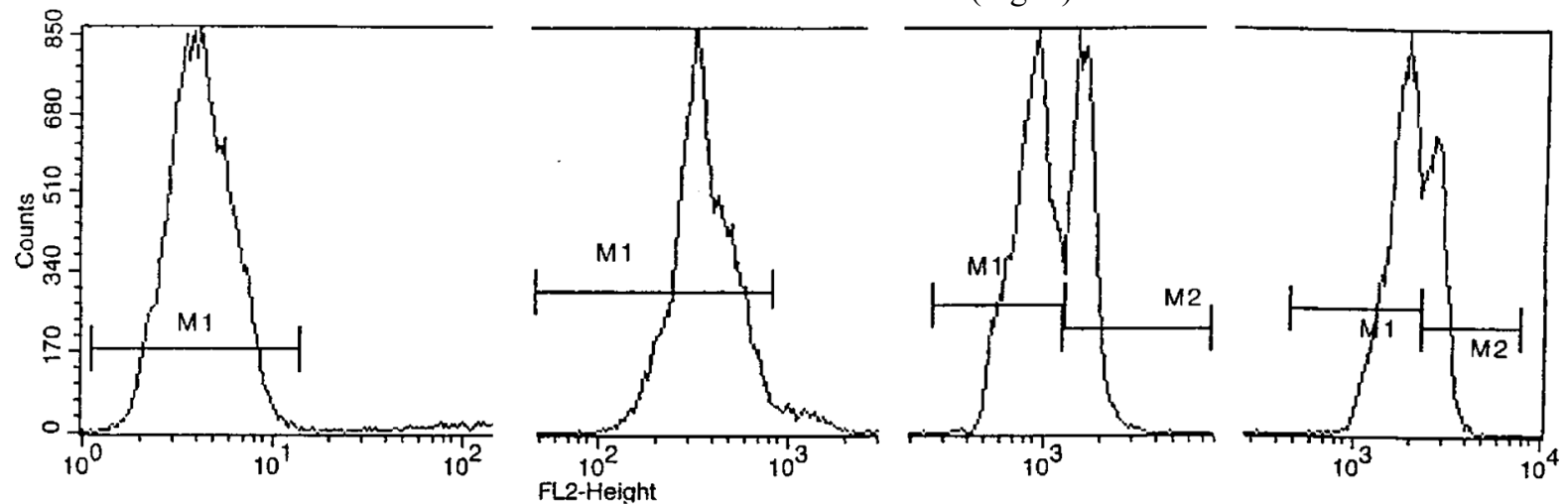

Fig. 1. Fluorescence histograms of HL-60 cells incubated with a fluorescently labeled Pluronic of progressively increasing concentrations; concentrations from left to right, $0.0002 ; 0.02 ; 0.2$, and $2 \%$. 

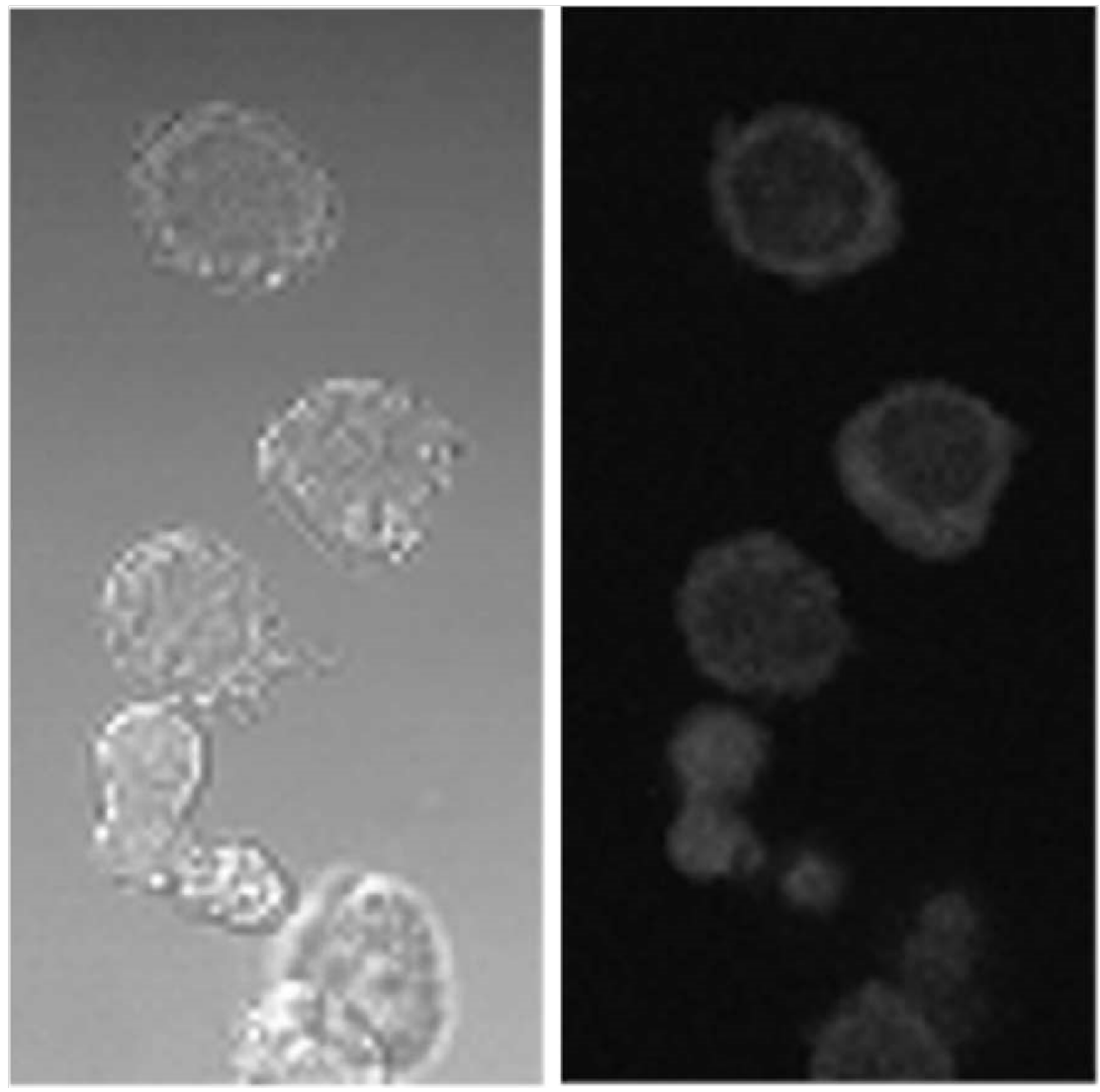

Fig. 2. Confocal images of HL-60 cells incubated with a fluorescently labeled Pluronic for $1 \mathrm{~h}$; incubation medium-PBS, Pluronic concentration $20 \mathrm{~g} / \mathrm{ml}$.

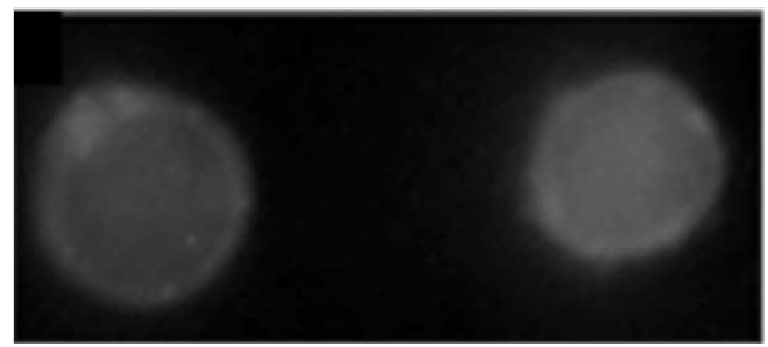

Fig. 3. Fluorescence micrographs of two HL-60 cells of the same sample incubated with a mixture of a labeled and unlabeled Pluronic P-105. The cells have distinctly different intracellular distribution of fluorescence. In the left cell, fluorescent molecules are localized in the extranuclear space, presumably in cytoplasmic vesicles; in the right cell, a release of the labeled molecules from the sequestering compartments is observed. Concentration of a fluorescently labeled Pluronic, $0.005 \%$; overall Pluronic concentration $1 \%$; incubation time, 10 min. 


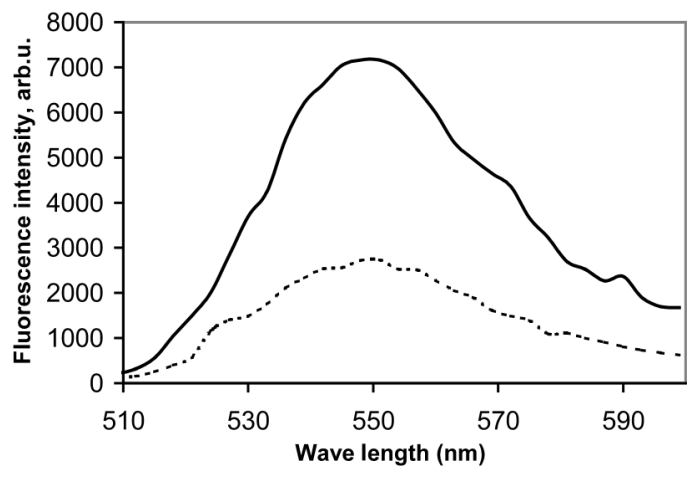

Fig. 4. Fluorescence spectra of a fluorescently labeled Pluronic P-105 at various $\mathrm{pH}$ values.

Note a bimodal character of fluorescence histograms at higher Pluronic concentrations (Fig. 1); in the fluorescence micrographs, this corresponds to the two populations of the cells with distinctly different distribution of fluorescence. Fig. 3 presents two cells of the same sample; the fluorescence of the left cell is relatively low and is confined to the extranuclear space; fluorescence of the right cell is much higher and is observed not only in the cytoplasm but also in the nucleus. Differences in Pluronic distribution at low and high Pluronic concentrations may be related to the differences in the mechanisms of the intracellular uptake, as explained below.

Fluorescence intensity of a fluorescent label used in this study was $\mathrm{pH}$ dependent and was significantly lower in the acidic than in the neutral environment (Fig. 4).

The intracellular uptake of Pluronic micelles is expected to proceed via fluid-phase endocytosis. In this case, micelles will be initially localized in the acidic environment of endosomes or lisosomes, where the fluorescence intensity of the label is low; the appearance of a peak with a higher fluorescence may be associated with permeabilization of acidic vesicles under the action of Pluronic micelles, resulting in a disruption of a $\mathrm{pH}$ gradient between the vesicles and the cytosol. This in turn would result in Pluronic release from the acidic vesicles, which allows Pluronic diffusing into cell nuclei. As indicated by a bimodal character of fluorescence histograms, only a fraction of cell population was affected by Pluronic micelles. In non-synchronized cell cultures, differences in the sensitivity of various cells in the population to the action of Pluronic micelles may be related to the cell age. In more details, these problems are discussed in a recent paper [25].

The intracellular uptake of Pluronic P-105 was observed not only at 37 but also at $4{ }^{\circ} \mathrm{C}$, when endocytosis was suppressed(Fig.5). Fig.5 indicates that more Pluronic was adsorbed to the surface and subsequently washed out from the surface when cells were incubated at $4{ }^{\circ} \mathrm{C}$ in comparison to $37{ }^{\circ} \mathrm{C}$ (compare fluorescence micrographs $\mathrm{A}$ and $\mathrm{B}$ for $37^{\circ} \mathrm{CtomicrographsCandDfor} 4^{\circ} \mathrm{C}$, before and after washing). However, Fig. 5 clearly shows that some fraction of Pluronic was internalized by the cells even at $4{ }^{\circ} \mathrm{C}$. As mentioned above, Pluronic P-105 does not form micelles at $4{ }^{\circ} \mathrm{C}$ but rather exists in the form of unimers [14]. The intracellular uptake of Pluronic at $4{ }^{\circ} \mathrm{C}$ indicated that Pluronic unimers entered the cells via simple diffusion through plasma membranes. Within the cells, Pluronic unimers internalized at $4{ }^{\circ} \mathrm{C}$ were confined to the extra nuclear cytoplasmic compartments(Fig. 5). Pluronic uptake was higher at 4 than at $37{ }^{\circ} \mathrm{C}$ as measured by the depletion from the incubation medium (Table 1); bimodal character of fluorescence histograms was observed only at $4{ }^{\circ} \mathrm{C}$ suggesting that in some fraction of the cells, Pluronic was internalized while in another fraction it was just adsorbed to the surface. The dependence of Pluronic uptake on the extracellular Pluronic concentration was similar at 4 and at $37{ }^{\circ} \mathrm{C}$. Also noteworthy is that at both temperatures, the discrepancy is observed between Pluronic uptake measured by depletion from the incubation medium and characterized by mean cell fluorescence; while the uptake measured by depletion drops, mean fluorescence of the cells goes through maximum with increasing concentration of the unlabeled Pluronic in the 
incubation medium (Table 1). We observed this discrepancy earlier in experiments with HL-60 and ovarian carcinoma cells A2780 drug-sensitive and drug-resistant cells; we interpreted it as resulting from differences in the fluorescence probe environment depending on the intracellular Pluronic concentration [25]. The data presented in Table 1 appear to confirm that even at $4{ }^{\circ} \mathrm{C}$, Pluronic intracellular concentration affects its compartmentalization.
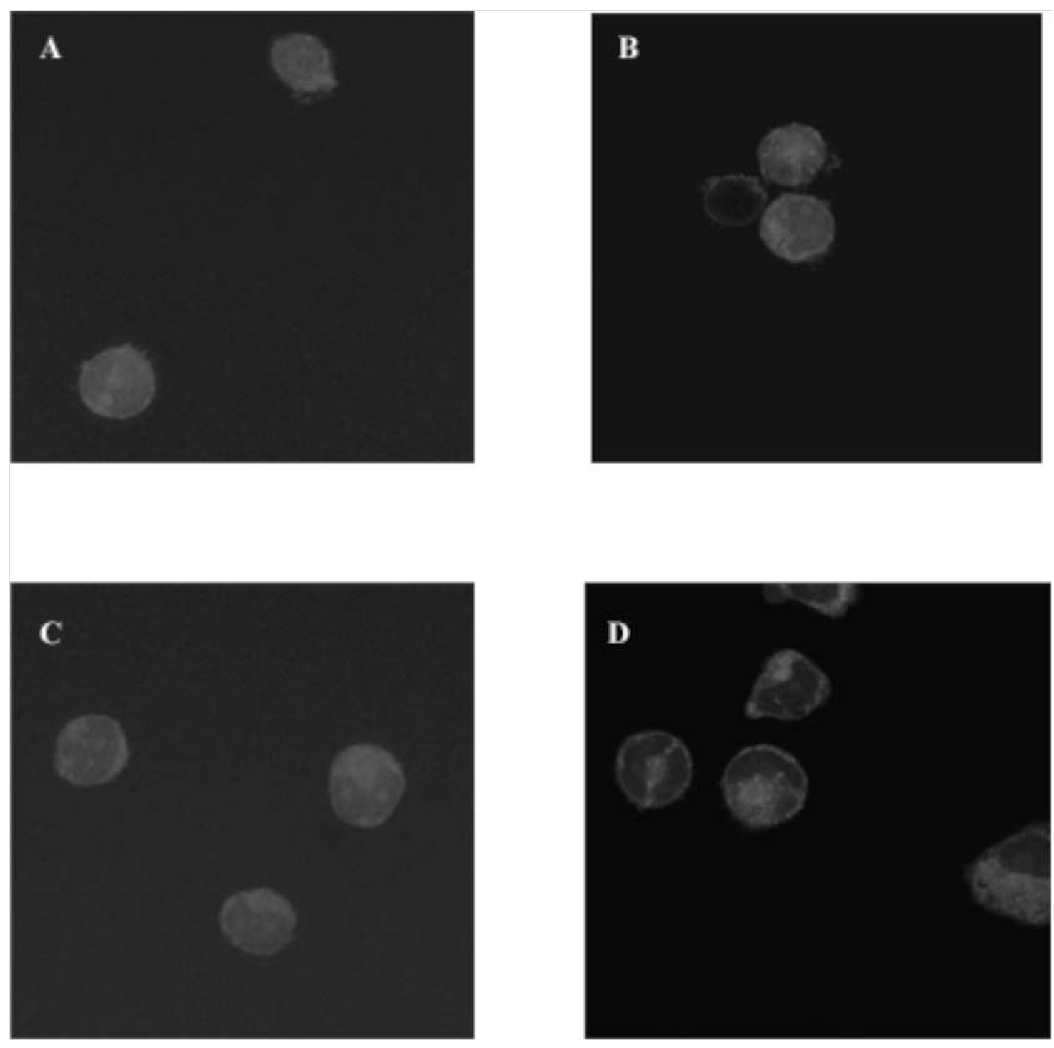

Fig. 5. Confocal images of HL-60 cells incubated with a fluorescently labeled Pluronic P-105 at $37{ }^{\circ} \mathrm{C}(\mathrm{A}$ and $\mathrm{B})$ and $4{ }^{\circ} \mathrm{C}(\mathrm{C}$ and $\mathrm{D})$ for 30 min before (A and C) and after (B and D) washing; incubation medium-PBS, Pluronic concentration $100 \mathrm{~g} / \mathrm{ml}$. The figure indicates that some fraction of a fluorescently labeled Pluronic is internalized by the cells even at $4{ }^{\circ} \mathrm{C}$.

Pluronic uptake at $4{ }^{\circ} \mathrm{C}$ was also reported in ref. [26] for two types of Pluronic, P-85 and L61; the hydrophobicity of the former is close to that of $\mathrm{P}-105$, while the second one is much more hydrophobic. Only minor differences in uptake at 4 and $37{ }^{\circ} \mathrm{C}$ were observed for Pluronic P-85, in accordance with our observations, while for L61 significantly higher uptake was observed at 37 than at $4{ }^{\circ} \mathrm{C}$. The authors concluded that at $4{ }^{\circ} \mathrm{C}$, Pluronic was adsorbed onto the cell surface, while at $37^{\circ} \mathrm{C}$ it was internalized via fluid-phase endocytosis. Based on the data of a present study, it is more likely that the uptake of Pluronic P-85 proceeded via diffusion of unimers through plasma membranes (note that Pluronic P-85 does 


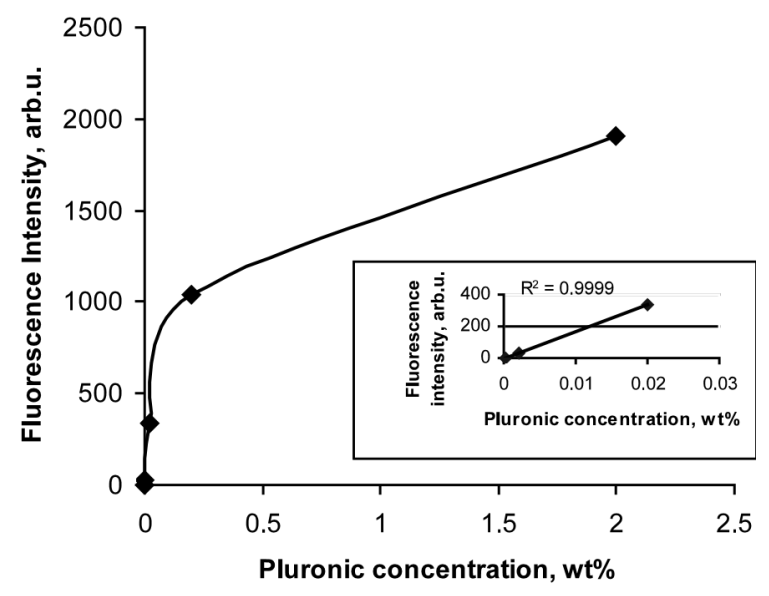

Fig. 6. Geometrical mean fluorescence intensity of the cells vs. concentration of a fluorescently labeled Pluronic in the incubation medium. At the low concentration range, fluorescence of the cells increases proportionally to the extracellular copolymer concentration (insert); proportionality is broken at higher polymer concentrations, presumably indicating a less efficient uptake of Pluronic micelles in comparison to unimers.

labeled Pluronic was more hydrophobic than the initial unlabeled copolymer; therefore, it is reasonable to assume that the $\mathrm{CMC}$ for the labeled copolymer was lower than that for the parental Pluronic P-105; we hypothesize that the break of proportionality was associated with the formation of micelles. This assumption was confirmed in experiments with the mixtures of the labeled and unlabeled Pluronic P-105 described below.

In the mixtures of the labeled and unlabeled Pluronic, the intracellular uptake of the labeled copolymer (at 37 ${ }^{\circ} \mathrm{C}$ ) decreased steadily with increasing concentration of the unlabeled Pluronic (Table 2). In these experiments, the intracellular uptake of the labeled Pluronic was measured by fluorescence depletion from the incubation medium; the initial concentration of the labeled Pluronic was the same in all experiments $(0.0016 \mathrm{wt} . \%)$. The overall Pluronic uptake was calculated according to Eq. (1). If we assume that the equilibrium is established between extracellular and not form micelles neither at 4 nor at $37^{\circ} \mathrm{C}$ at the concentrations used in ref. [26]);

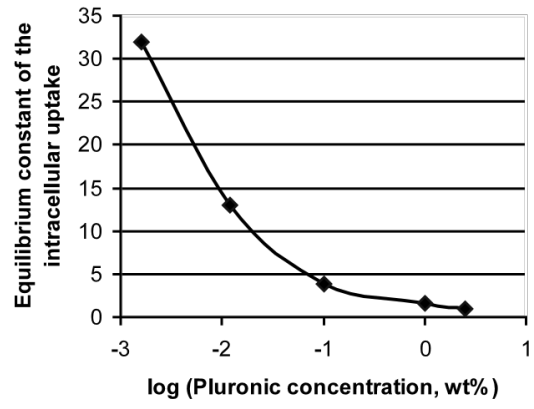

Fig. 7. Partition coefficient of Pluronic P-105 between the cell and extracellular medium vs. Pluronic concentration in the extracellular medium.

however, Pluronic L61 forms micelles at $37^{\circ} \mathrm{C}$ and most probably was internalized via endocytosis.

Pluronic uptake at $37^{\circ} \mathrm{C}$ was observed over the whole concentration range studied, from 0.0002 to $10.0 \mathrm{wt} . \%$. At a concentration range of $0.0002-0.02 \mathrm{wt} . \%$, the fluorescence was roughly proportional to Pluronic concentration in the incubation medium; however, the proportionality broke sharply when Pluronic concentration increased above $0.02 \%$ (Fig. 6). Note that a fluorescently internalized Pluronic molecules at all Pluronic concentrations (which is not necessarily true for Pluronic micelles), the data of Table 2 allow estimating partition 
coefficient of Pluronic internalization, $K_{\text {eq. }}$ As shown in Table 2, the equilibrium constant of Pluronic internalization dropped more than 40 -fold upon transition from Pluronic unimers $(0.0016 \mathrm{wt} \%$ ) to Pluronic micelles $(2.5 \mathrm{wt} . \%)$. In the intermediate concentration range between unimers and micelles, Pluronic forms loose water penetrated aggregates [24]. The data of Table 2 indicate that the intracellular uptake of aggregates is less favorable than that of unimers. It is reasonable to assume that the uptake of the aggregates proceeds via detachment of the unimers, which requires additional energy and, therefore, is less effective. The dependence of the partition coefficient of Pluronic on the extracellular Pluronic concentration is shown in Fig. 7. Based on the data presented above, the shielding effect of Pluronic micelles on the intracellular uptake of drug can be explained. In what follows, we assume that in micellar Pluronic solutions, drug molecules enter the cells only inside Pluronic micelles that are internalized via endocytosis. In contrast, in solutions of Pluronic unimers, drug enters the cells in association with Pluronic unimers internalized via a simple diffusion (this is assumed for generality; in a real situation, drug is probably internalized independently on Pluronic unimers). In a Pluronic P-105 solution of 0.001 wt.\% comprising $50 \mathrm{~g} / \mathrm{ml}$ drug with a molecular weight of $550 \mathrm{Da}$ (which is a good approximation for most anthracyclin drugs), each Pluronic molecule is 'associated' with 60 drug molecules. In a 1 wt.\% micellar Pluronic solution, assuming a complete micellization and an aggregation number of 100 , the concentration of micelles is $1.5 \times 10^{-5} \mathrm{M}$; for the same drug concentration as above, each micelle carries in average six drug molecules. Based on the values of equilibrium constant presented in Table 2 , the uptake of micelles from a $1 \mathrm{wt} . \%$ Pluronic solution would be about $70 \%$ of that of the unimers from a $0.001 \mathrm{wt} . \%$ solution; therefore, the uptake of drug from a micellar solution is about $7 \%$ of that from a unimeric solution.

We need to emphasize that the above calculation is presented here exclusively for the sake of illustration; a real situation is much more complicated and depends on the drug/carrier and drug/ cell interaction in a particular system.

At all Pluronic concentrations, application of ultrasound enhanced both the intracellular Pluronic uptake and Pluronic release from confining compartments $[18,19]$. The increased uptake of Pluronic micelles induced by ultrasound irradiation results in the increased uptake of micelles are encapsulated drugs $[18,19]$.

\section{Conclusions}

Both Pluronic P-105 unimers and micelles were internalized by HL-60 cells. The uptake of micelles was less efficient than that of the unimers. The unimers were presumably internalized via diffusion through cell membranes while micelles were internalized via fluid-phase endocytosis.

\section{Acknowledgements}

This work was supported by the NIH grant R01 CA76562-01A1.

\section{References}

[1] N. Rapoport, Coll. Surf. B Biointerf. 3 (1999) 93-111.

[2] N. Rapoport, N. Munshi, L. Pitina, W.G. Pitt, Polym. Preprints 38 (1997) 620-621.

[3] N. Rapoport, L. Pitina, J. Pharm. Sci. 87 (1998) 321-325.

[4] N. Munshi, N. Rapoport, W.G. Pitt, Cancer Lett. 118 (1997) 13-19.

[5] N.Y. Rapoport, J.N. Herron, W.G. Pitt, L. Pitina, J. Control. Release 58 (1999) 153-162.

[6] G.A. Husseini, G.D. Myrup, W.G. Pitt, D.A. Christensen, N.Y. Rapoport, J. Control. Release 69 (2000) $43-52$.

[7] G.S. Kwon, K. Kataoka, Adv. Drug Deliv. Rev. 16 (1995) 295-309. 
[8] B.G. Yu, T. Okano, K. Kataoka, G. Kwon, J. Control. Release 53 (1998) 131-136.

[9] V.Y.u. Alakhov, E.Y. Moskaleva, E.V. Batrakova, A.V. Kabanov, Bioconjugate Chem. 7 (1996) $209-216$.

[10] A. Venne, S. Li, R. Mandeville, A. Kabanov, V. Alakhov, Cancer Res. 56 (1996) 3626-3629.

[11] E.V. Batrakova, S. Li, D.W. Miller, A.V. Kabanov, Pharm. Res. 16 (1999) 1366-1372.

[12] E.V. Batrakova, S. Lee, S. Li, A. Venne, V. Alakhov, A.V. Kabanov, Pharm. Res. 16 (1999) 1373-1379.

[13] K.E. Uhrich, S.M. Cannizzaro, R.S. Langer, K.M. Shakesheff, Chem. Rev. 99 (1999) 3181-3198.

[14] P. Alexandridis, J.F. Holzwarth, T.A. Hatton, Macromolecules 27 (1994) 2414-2425.

[15] H. Maeda, L.M. Seymour, Y. Miyamoto, Bioconjugate Chem. 3 (1992) 351-362.

[16] G.S. Kwon, K. Kataoka, Adv. Drug Deliv. Rev. 16 (1995) 295-309.

[17] K. Kataoka, T. Matsumoto, M. Yokoyama, T. Okano, Y. Sakurai, S. Fukushima, K. Okamoto, G.S. Kwon, J. Control. Release 64 (2000) $143-153$.

[18] A. Marin, M.D. Muniruzzaman, N. Rapoport, J. Control. Release 71 (2001) 239-249.

[19] A. Marin, M.D. Muniruzzaman, N. Rapoport, J. Control. Release 75 (2001) 69-81.

[20] M.D. Bednarski, J.W. Lee, M.R. Callstrom, K.C. Li, Radiology 204 (1997) 263-268.

[21] N.Y. Rapoport, A.I. Smirnov, A.I. Timoshin, A.M. Pratt, W.G. Pitt, Arch. Biochem. Biophys. 344 (1997) 114-124. [22] K. Tachibana, T. Uchida, K. Ogawa, N. Yamashita, K. Tamura, Lancet 353 (1999) 1409.

[23] J. Liu, T.N. Lewis, M.R. Prausnitz, Pharm. Res. 15 (1988) 918-924.

[24] N. Rapoport, K. Caldwell, Coll. Surf. B Biointerf. 3 (1994) 217-228.

[25] N. Rapoport, A. Marin, Md. Muniruzzaman, J. Pharm. Sci. 91 (2002) 157-170.

[26] N.S. Melik-Nubarov, O.O. Pomaz, T.Y.u. Dorodnych,

S.A. Arzhakov, FEBS Lett. 446 (1999) 194-198.

G.A. Badun, A.L. Ksenofontov, O.B. Schemchukova, 